\title{
Sensitivity for Heavy Resonances at the High- Luminosity LHC with the Phase-2 CMS Detector
}

\author{
Kerstin Hoepfner ${ }^{1}$ \\ On behalf of the CMS Collaboration \\ RWTH Aachen, III. Phys. Inst. A \\ Otto-Blumenthal-Str., Aachen, Germany \\ E-mail: kerstin.hoepfnerecern.ch
}

To extend the LHC physics program, it is foreseen to operate the LHC in the future with an unprecedented high luminosity. To maintain the experiment's physics potential in the harsh environment of this so-called phase-2, the detector will be upgraded. At the same time the detector acceptance will be extended and new features such as a L1 track trigger will be implemented. Simulation studies evaluated the physics reach of benchmark searches for physics beyond the SM. One of the open question being discussed right now, are models explaining the observed flavour anomalies. They postulate leptoquarks or Zprime-like new bosons, with enhanced couplings to third generation particles. In this context future searches in the ttbar mass spectrum are discussed, as well as searches for leptoquarks and heavy bosons with tau in the final state. Another class of interesting models suggest compositeness, a fermion substructure, leading to a potential observation of excited leptons or composite neutrinos. All these studies show a significantly enhanced sensitivity and will shape the future research program.

European Physical Society Conference on High Energy Physics - EPS-HEP2019 -

10-17 July, 2019

Ghent, Belgium

\footnotetext{
${ }^{1}$ Speaker

(C) Copyright owned by the author(s) under the terms of the Creative Commons

Attribution-NonCommercial-NoDerivatives 4.0 International License (CC BY-NC-ND 4.0).
} 


\section{Upgrade of LHC and CMS provide great physics opportunities}

The LHC proton-proton collider now operates for almost ten years, at present with a center-ofmass energy of $13 \mathrm{TeV}$. The so-called phase-1 should last till 2023 allowing the multi-purpose experiments to collect an expected integrated luminosity of $300 / \mathrm{fb}$. This phase- 1 will be followed by a long shutdown during which accelerator and experiments are being upgraded for ten times more integrated luminosity during phase-2, the so-called high-luminosity LHC (HLLHC). Such a large statistics data set will provide great physics opportunities: another new particle may be discovered (e.g. dark matter, heavy resonances, but one should also be prepare for „unexpected“ new physics), and processes with weak couplings become accessible. In case, signs of new physics are seen, its properties such as particle mass, couplings, spin, etc. are to be measured. The HL phase- 2 maybe followed by another phase with higher collision energy, the so-called HE-LHC. As a benchmark $27 \mathrm{TeV}$ are studied with $15 \mathrm{ab}^{-1}$ of integrated luminosity.

This report showcases a few examples of CMS physics studies for the HL-LHC. More results can be found in [6]. The presented physics studies are either carried out with the parametrized event simulation DELPHES [7], or are projections of Run-2 exclusion limits to $3000 \mathrm{fb}^{-1}$. The impact of our future knowledge of systematic uncertainties on the physics reach is also considered e.g. expected improvements on theoretical knowledge of higher order corrections, while detector understanding and data-driven methods will profit from larger statistics.

\section{Search for ttbar resonances at HL/HE}

Among the models predicting a ttbar resonance, are extra dimension models suggesting a spin-2 RS graviton. Such graviton may have enhanced couplings to $3^{\text {rd }}$ generation SM particles and hence be first seen when scanning die ttbar invariant mass spectrum. Fig.1 shows these distributions for the $3 \mathrm{ab}^{-1}$ of HL-LHC (left) and $15 \mathrm{ab}^{-1}$ for HE-LHC (right) [1]. With HL, the 5 -sigma discovery sensitivity reaches $\approx 6 \mathrm{TeV}$ of mass for the new particle when combining all possible top decay channels (semi-leptonic and hadronic) and applying $b$ - and t-tagging. The sensitvity increases to $10 \mathrm{TeV}$ masses at HE-LHC for the same channel combination. This analysis requires substructure techniques for boosted jets.
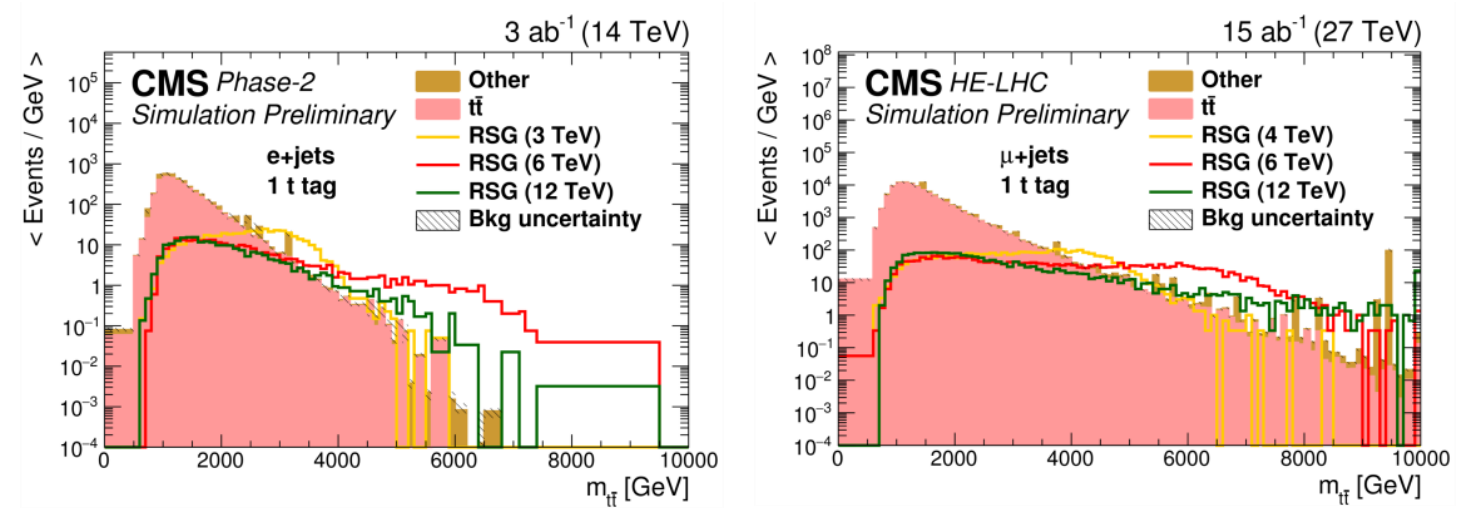

Fig. 1: Signal and background for an RS graviton for three given masses. On the left for the e+jets class at HL-LHC with $14 \mathrm{TeV}$. On the right for the mu+jets class at HE-LHC with $27 \mathrm{TeV}$ [1]. 


\section{Search for compositeness}

Compositeness would yield excited leptons, such as $\mathrm{e}^{*}$ or $\mu^{*}$, being produced in association with the corresponding SM lepton partner. Different final states are possible following either a gauge (emission of a photon, $\mathrm{Z}$ or $\mathrm{W}$ ) of contact interaction decay. The classical search looks for final states of two electrons or muons and a photon. The process is steered by two model parameters: the excited lepton mass $\mathrm{m}\left(\ell^{*}\right)$ and the compositeness scale $\Lambda$. To extract a potential ee $\gamma$ or $\mu \mu \gamma$ signal, $\mathrm{m}^{\mathrm{min}}$ and $\mathrm{m}^{\mathrm{max}}$ are reconstructed by combining either of the two leptons with the photon. When combining both to a 2-dimensional plane, as seen in Fig.2-left, the signal looks like an inverted $\mathrm{L}$ (see signal for an $\mathrm{e}^{*}$ of $3.5 \mathrm{TeV}$ mass in yellow). This signal can be well separated from SM background (shown in blue in Fig.2-left) [4]. The expected 5-sigma discovery significance at HL-LHC corresponds to $5.1 \mathrm{TeV}$ in $\ell^{*}$ mass (Fig.2-right), while the exclusion limit would be around $5.8 \mathrm{TeV}$, to be compared to the present limit of $3.8 \mathrm{TeV}$ from analyzing $36 \mathrm{fb}^{-1}$ of phase-1 data.
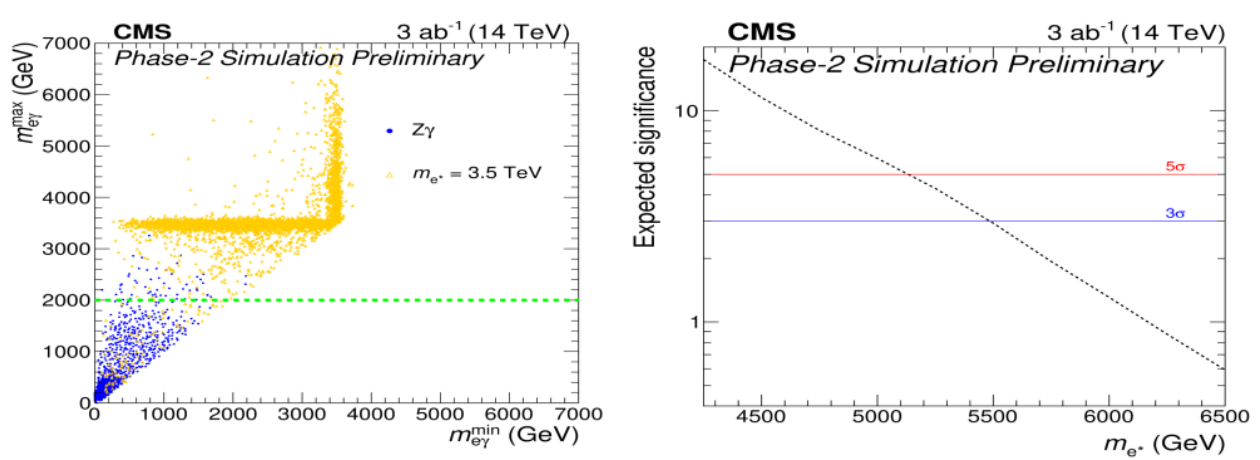

Fig. 2: Left: The signal of an excited lepton will manifest itself as an inverted L (yellow) in the 2D space of $M^{\text {max }}$ and $M^{\text {min }}$ [4]. Right: Expected discovery significance with $3 a b^{-1}$ shown here for excited electrons. Excited Muons are comparable.

Compositeness could also yield a heavy composite majorana neutrino $\left(\mathrm{N}_{\ell}\right)$ detectable through decay modes such as $\mathrm{qq} \rightarrow \ell \mathrm{N}_{\ell} \rightarrow$ eejj/ $\mu \mu \mathrm{jj}$. The two jets are reconstructed as a fat jet (J). Similar to above, the model parameters are the heavy neutrino mass $\mathrm{M}\left(\mathrm{N}_{\ell}\right)$ and the compositeness scale $\Lambda$. The distribution of the discriminating variable $M(\ell \ell J)$, created by two high $\mathrm{p}_{\mathrm{T}}$ leptons and a fat jet $(\mathrm{J})$, is seen in Fig.3-left. The exclusion, either electron or muon channel, for $M\left(\mathrm{~N}_{\ell}\right)=\Lambda$ improves from presently $4.6 \mathrm{TeV}$ to $8 \mathrm{TeV}$ at HL-LHC and $12 \mathrm{TeV}$ at HE-LHC, respectively, while the discovery potential corresponds to $7.5 \mathrm{TeV}$ (HL) and $11.5 \mathrm{TeV}$ (HE), respectively [2].
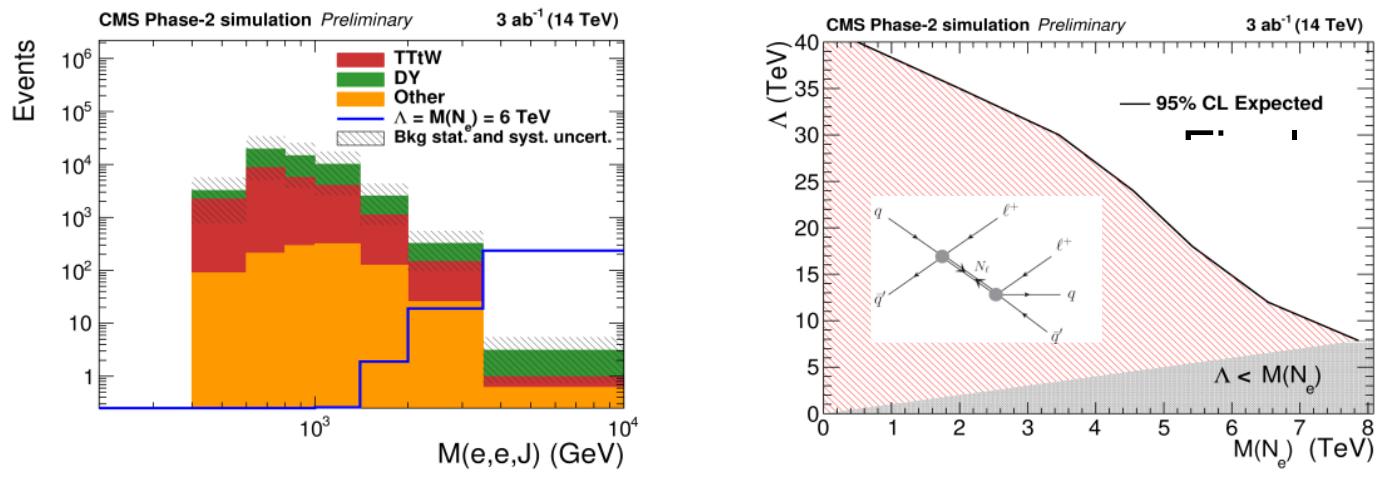

Fig. 3: Left: Search for a heavy composite majorana neutrino[2]. Signal and backgrounds are shown. Right: Exclusion limit in terms of compositeness scale $\Lambda$ and mass of the heavy neutrino. 


\section{Opportunities with 3rd generation particles in the final state}

Flavour observations might be explained by the existence of leptoquarks (LQ) or new heavy bosons, both with enhanced couplings to third generation SM particles, motivating searches such as $\mathrm{W}^{\prime} \rightarrow \tau \nu$. Fig.4-left depicts the transverse mass $\left(\mathrm{m}_{\mathrm{T}}\right)$ distribution for a $\mathrm{W}^{\prime}[5]$ search built by the hadronically decaying tau and missing transverse momentum due to the non-observed neutrino. At HL-LHC W' bosons could be discovered in this channel up to $6.5 \mathrm{TeV}$ in mass or excluded up to $7 \mathrm{TeV}$ [5]. A model-independent cross section limit for a $\tau \mathrm{v}$ final state shows Fig.4-right.
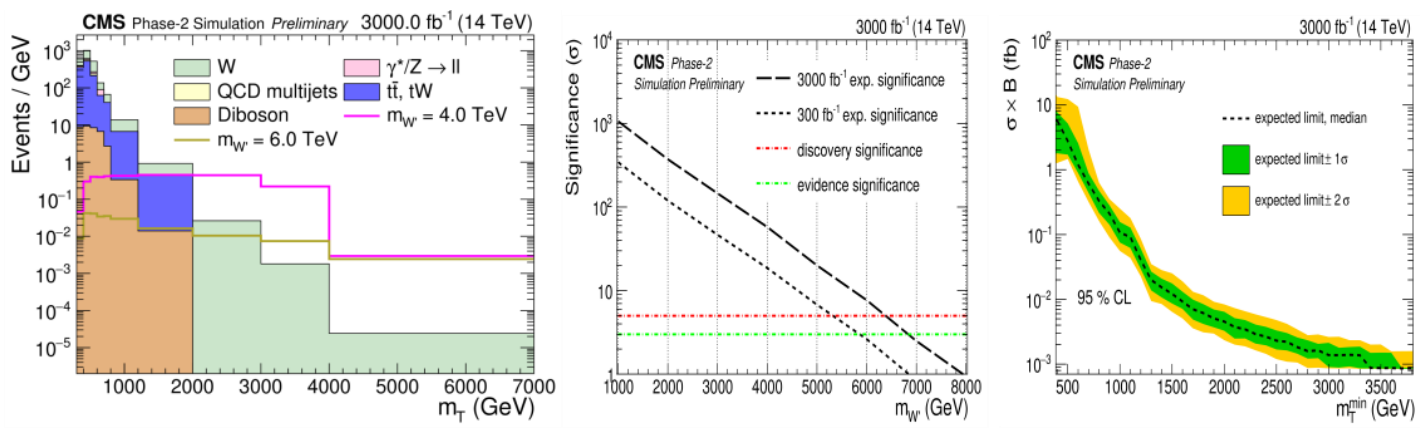

Fig.4: Left: Transverse mass distribution $\left(m_{T}\right)$ with signal examples and background for a $W^{\prime} \rightarrow \tau v$ search. Middle: Expected significance with $3 a b^{-1}$. Right: Model-independent cross-section limit for $\tau v$ final states [5].

LQ can be produced in pairs or singly (see Fig.5), where the latter becomes dominant for large $\lambda$, while pair-production dominates the phase space of low $\lambda$ and high LQ masses. With couplings to the $3^{\text {rd }}$ generation, the final state contains b-tagged jets and tau leptons being reconstructed as jets from the hadronic tau decay. The discovery significance for both production channels is shown in the table for two uncertainty scenarios [3]. Fig.5-right compares the expected sensitivity to the preferred parameter space (blue hatched area) in $\lambda$ and $\mathrm{M}_{\mathrm{LQ}}$ compatible with the flavor observations [3].

\begin{tabular}{|c|c|c|c|}
\hline \multirow{2}{*}{ Production channel } & \multirow{2}{*}{$\begin{array}{c}\text { Uncertainty } \\
\text { scenario }\end{array}$} & \multicolumn{2}{|c|}{ LQ mass [GeV] } \\
\hline & & $300 \mathrm{fb}^{-1}$ & $3000 \mathrm{fb}^{-1}$ \\
\hline \multirow{2}{*}{ single LQ } & stat. only & 784 & 1135 \\
\hline & stat. + syst. & 732 & 1130 \\
\hline \multirow{2}{*}{ LQ pair } & stat. only & 1253 & 1520 \\
\hline & stat. + syst. & 1249 & 1518 \\
\hline
\end{tabular}

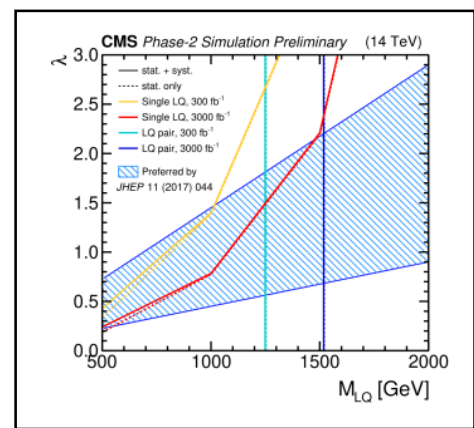

Fig.5: Search for LQ, produced either singly or in pairs. The table shows the expected limits. Right: The sensitivity in comparison to the region (blue) favored by flavor observations [3].

\section{References}

[1] CMS-PAS-FTR-18-009 [2] CMS-PAS-FTR-18-006 [3] CMS-PAS-FTR-18-028

[4] CMS-PAS-FTR-18-029 [5] CMS-PAS-FTR-18-030

[6] arXiv:1812.07831 (Yellow report) [7] DELPHES simulation, arXiv:1307.6346 